\title{
The Co-occurrence of NDM-5, MCR-1, and FosA3-Encoding Plasmids Contributed to the Generation of Extensively Drug-Resistant Klebsiella pneumoniae
}

OPEN ACCESS

Edited by:

Che-Hsin Lee,

National Sun Yat-sen University,

Taiwan

Reviewed by:

Prasanth Manohar,

Zhejiang University-University

of Edinburgh Institute, China

Abinaya Badri,

Ginkgo BioWorks, United States

*Correspondence:

Fangyou Yu

wzjxyfy@163.com

${ }^{\dagger}$ These authors have contributed equally to this work

Specialty section:

This article was submitted to

Antimicrobials, Resistance

and Chemotherapy,

a section of the journal

Frontiers in Microbiology

Received: 08 November 2021

Accepted: 30 November 2021

Published: 03 January 2022

Citation:

Zhou Y, Ai W, Cao Y, Guo Y, Wu X,

Wang $B$, Rao $L, X u Y$, Zhao $H$,

Wang $X$ and Yu $F$ (2022) The

Co-occurrence of NDM-5, MCR-1,

and FosA3-Encoding Plasmids

Contributed to the Generation

of Extensively Drug-Resistant

Klebsiella pneumoniae.

Front. Microbiol. 12:811263.

doi: 10.3389/fmicb.2021.811263
Ying Zhou't, Wenxiu Ai2t, Yanhua Cao ${ }^{3 t}$, Yinjuan Guo ${ }^{1}$, Xiaocui Wu', Bingjie Wang', Lulin Rao ${ }^{1}$, Yanlei Xu' ${ }^{1}$, Huilin Zhao ${ }^{1}$, Xinyi Wang ${ }^{1}$ and Fangyou Yu ${ }^{4 *}$

${ }^{1}$ Department of Clinical Laboratory Medicine, Shanghai Pulmonary Hospital, School of Medicine, Tongji University, Shanghai, China, ${ }^{2}$ Department of Respiratory Medicine, The First Affiliated Hospital of Wenzhou Medical University, Wenzhou, China,

${ }^{3}$ Department of Respiratory Intensive Care Unit, Shanghai Pulmonary Hospital, School of Medicine, Tongji University, Shanghai, China, ${ }^{4}$ Department of Laboratory Medicine, The First Affiliated Hospital of Wenzhou Medical University,

Wenzhou, China

The rise and global dissemination of extensively drug-resistant (XDR) bacteria are often related to plasmid-borne mobile antimicrobial resistance genes. Notably, isolates having multiple plasmids are often highly resistant to almost all the antibiotics available. In this study, we characterized an extensively drug-resistant Klebsiella pneumoniae 1678, which exhibited high-level resistance to almost all the available antibiotics. Through whole-genome sequencing (WGS), more than 20 resistant elements and 5 resistant plasmids were observed. Notably, the tigecycline resistance of $K$. pneumoniae 1678 was not related to the plasmid-borne tet $A$ gene but associated with the overexpression of $A c r A B$ and OqxAB efflux pumps, according to the susceptibility results of tetAtransformant and the related mRNA quantification of RND efflux pumps. Except for tigecycline resistance, three plasmids, mediating resistance to colistin, Fosfomycin, and ceftazidime-avibactam, respectively, were focused. Detailed comparative genetic analysis showed that all these plasmids belonged to dominated epidemic plasmids, and harbored completed conjugation systems. Results of conjugation assay indicated that these three plasmids not only could transfer to E. coli J53 with high conjugation frequencies, respectively, but also could co-transfer to $E$. coli J53 effectively, which was additionally confirmed by the S1-PFGE plasmids profile. Moreover, multiple insertion sequences (IS) and transposons (Tn) were also found surrounding the vital resistant genes, which may form several novel mechanisms involved in the resistant determinants' mobilization. Overall, we characterized and reported the uncommon co-existence and co-transferring of FosA3-, NDM-5, and MCR-1-encoding plasmids in a K. pneumoniae isolate, which may increase the risk of spread of these resistant phenotypes and needing great concern.

Keywords: $K$. pneumoniae, plasmid, fosA3, bla $a_{\mathrm{NDM}-5}, \mathrm{mcr-1}$, mobile elements 


\section{INTRODUCTION}

Carbapenem-resistant Klebsiella pneumoniae (CRKP) has recently emerged as a major class of bacterial pathogens that pose a significant threat to global public health, since it can cause high-fatal infections, and the treatment choices are very limited (Chen et al., 2014). The emergence of antibiotic resistance arises the development of several new antibiotics, such as tigecycline (Chopra, 2002) and ceftazidime-avibactam (Zhanel et al., 2013), and the re-evaluation of old antibiotics such as Fosfomycin and polymyxin as a potential regimen for treating such multidrugresistant bacteria (Doi, 2019). Tigecycline, colistin, Fosfomycin, and ceftazidime-avibactam were considered as the most effective agents for the CRKP infection treatments, and were even regarded as the last "trump card" to defend against CRKP (Doi, 2019). However, clinical isolates resistant to those four antibiotics emerged frequently (Petrosillo et al., 2019; Fang et al., 2020; Yahav et al., 2020; Zurfluh et al., 2020; Yusuf et al., 2021). Hence, verifying the related mechanism and demonstrating the potential of the spread of these resistant phenotypes in clinical isolate are urgent, which are the vital clues to solve antibiotic resistance.

The acquisition of antibiotic resistance was always associated with mobile genetic elements (MGEs) such as conjugative and mobilizable plasmids and transposons (Partridge et al., 2018). In Enterobacteriaceae, Fosfomycin-modifying enzymes are the important factors to inactivate the Fosfomycin, genes encoding these enzymes (fos $A$ ) are frequently found on plasmids, transposons, or within integrons (Zurfluh et al., 2020). Lipopolysaccharide modifications are the key issues to reduce the antibiotic effect of polymyxin. In addition to the two-component systems (TCSs) PhoP/PhoQ, PmrA/PmrB, and CrrA/CrrB (Mcconville et al., 2020), the plasmid-mediated mor genes (such as $m c r-1$ ) mediated enzymes are the most noteworthy way to modify lipopolysaccharides, which not only result in polymyxin resistance, but also result in the transferring of this antibiotic resistant-phenomena worldwide (Xiaomin et al., 2020). The tigecycline resistance is sometimes associated with the overexpression of the efflux pumps AcrAB and OqxAB (Bialek-Davenet et al., 2015). Meanwhile, mutations in tetracycline resistance factors, including efflux pumps (tet $A$, tetB, and tetK) (Foong et al., 2020; Xu et al., 2021) and other plasmid-borne tigecycline resistance genes, tet $(X)$ (Sun et al., 2019) and tmexCD-toprJ (Hirabayashi et al., 2021), have also been reported to contribute to $K$. pneumoniae resistance to tigecycline. Although ceftazidime-avibactam (CAZ/AVI) exhibited remarkable inhibition to KPC carbapenemase, it is not active against Metallo- $\beta$-Lactamases (MBL)-producing bacteria, such as bla $a_{N D M}$-positive isolates (Yang et al., 2020). Overall, since almost all the antibiotic resistance could be carried by various MGEs, once the bacteria obtain multiple resistant elements simultaneously, they would become resistant to those antibiotic agents, and the therapeutic options would be very limited.

The co-occurrence of multiple resistant plasmids in one isolate often results in the resistance to almost all available antibiotics, and also promotes the dissemination of resistance determinants. Several studies also reported some co-existence of resistant-genes in Enterobacteriaceae, like fos 3 and bla $\mathrm{KPC}-2_{2}$ (Tang et al., 2020), or $m c r-1$ and $b l a_{\mathrm{NDM}-5}$ (Sun et al., 2016), these co-existences make the strain become extensively drug-resistant to multiple antibiotics. Notably, although there are several reports about the co-existence of $m c r-1$ and $b l a_{\mathrm{NDM}-5}$ in one plasmid or two separated plasmids, most of these plasmids were harbored by $E$. coli strains of animal origin or environmental origin, which is uncommon in K. pneumoniae (Yang et al., 2016; Quan et al., 2017; Mao et al., 2018; Wang et al., 2018, 2021; Chen et al., 2019; Liu and Song, 2019; Han et al., 2020; Yuan et al., 2021).

In this study, our aim was to characterize an XDR K. pneumoniae isolated from a clinical patient, which is not only highly resistant to carbapenems, but also resistant to all the alternative antibiotics, including tigecycline, ceftazidimeavibactam, Fosfomycin, and polymyxin. We applied wholegenome-sequencing (WGS) to explore the potential molecular mechanisms mediating this multidrug-resistance, and observed three key resistant plasmids. We also made a detailed analysis of the plasmid-backbone and the conjugation region to evaluate the potential movability, and applied the conjugation assay to further determine the dissemination risk of these resistant determinants. In addition to the plasmids, we described other related MEGs through the genetic comparisons as well. Overall, our goal was to report and describe a clinical multi-drug resistant K. pneumoniae clearly, and emphasize the possible risk of these strains.

\section{MATERIALS AND METHODS}

\section{Bacterial Strains}

To explore the molecular epidemic feature of carbapenemresistant $K$. pneumoniae in China mainland, we randomly collected 137 carbapenem-resistant K. pneumoniae isolates from blood samples of individual patients at nine hospitals in eight Chinese provinces, from January 2015 to December 2018. The isolates were cultivated with LB medium. We applied WGS to analyze the presence of resistance elements among these isolates, and observed $K$. pneumoniae strain 1678 co-harboring multiple resistance determinants including fos $A 3, m c r-1$, and $b l a_{\mathrm{NDM}-5}$, that were uncommon in other K. pneumoniae. K. pneumoniae strain 1678 was isolated from the blood samples of a 71-year-old patient in 2018, in a tertiary hospital in Shanghai, China. Plasmid transformation and conjugation were performed with Escherichia coli TOP10 and J53 (sodium-azide ${ }^{\mathrm{R}}$ ) used as recipients for the selection of tet $A-$, fosA3-, bla $a_{\mathrm{NDM}-5}$, or $m c r-1$-positive transformants and related transconjugants, respectively.

\section{Antimicrobial Susceptibility Test}

The minimum inhibitory concentration (MIC) of the original isolate 1678 and all the transformants and transconjugants were determined by both broth microdilution and the polymyxin MIC was determined by the E-test methods following the Clinical and Laboratory Standards Institute guidelines. Briefly, for the broth microdilution and agar dilution method, pick 12 bacterial clones diluted with saline to $0.5 \mathrm{McF}$, and then dilute such bacterial suspension to $0.5 \times 10^{-2} \mathrm{McF}$ with $\mathrm{CAMHB}$ broth. The cells were inoculated in prefabricated commercial 96-well antibiotic culture plates or antibiotic agars, $100 \mu \mathrm{L}$ 
per well, and incubated overnight for $18 \mathrm{~h}$ at $37^{\circ} \mathrm{C}$. E-test method using a colistin strip (concentration range, 0.016$256 \mu \mathrm{g} / \mathrm{ml}$ ) (bioMérieux) was performed with Mueller-Hinton agar (MHA) (BD) plates in accordance with recommendations of the manufacturers. Notably, the Fosfomycin MIC was tested by the agar dilution using agar media supplemented with $25 \mu \mathrm{g} / \mathrm{mL}$ of glucose-6- phosphate. Escherichia coli ATCC25922 was used as a quality control strain for MIC determination. The interpretative breakpoints were based on CLSI2021 (Clinical and Laboratory Standards Clinical and Laboratory Standards Institute, 2021a; Clinical and Laboratory Standards Clinical and Laboratory Standards Institute, 2021b).

\section{Quantitation of mRNA Expression}

To explore whether the tigecycline-resistant phenotype was related with the overexpression of $A c r A B$ and OqxAB efflux pumps, we applied q-RT-PCR (quantitative-real-time-PCR) to measure the relative gene expression. All the primers were listed in Supplementary Table 1. RNA manipulation and real-time PCR were performed as described previously (Zheng et al., 2018). All bacterial samples were cultured in LB medium that did not contain any antibiotics. RNA was isolated as per the protocol of the MiniBEST Universal RNA extraction kit (TaKaRa, Tokyo, Japan). RNA samples for real-time PCR were pre-treated with DNase I (TaKaRa, Tokyo, Japan). Real-time PCR was conducted on a 7,500 system (Applied Biosystems, Foster City, CA, United States) using SYBR Premix ExTag (Takara, Tokyo, Japan). The expression of target genes was standardized relative to the $16 \mathrm{~S}$ rRNA housekeeping gene $\operatorname{rrs} E$. The expression levels of the target genes were compared with those of K. pneumoniae ATCC 13,883 (tigecycline susceptible). The relative expression levels of genes were calculated using the $\Delta \triangle C T$ method. All assays were performed in triplicate with three independent RNA preparations.

\section{Whole Genome Sequencing and Bioinformatics Analysis}

The genomic DNA of 1678 was extracted using a commercial DNA extraction kit (Qiagen, Germany) and was sequenced using short- and long-read massively parallel sequencing. The paired-end short Illumina reads were used to correct the long PacBio reads utilizing proovread, and then the corrected PacBio reads were assembled de novo utilizing SMARTdenovo ${ }^{1}$. Resistant plasmid replicons were identified using the PlasmidFinder database using the minimum coverage and minimum identities of $90 \%{ }^{2}$. Acquired antibiotic resistance genes were identified using ResFinder $^{3}$ with the default threshold. To determine whether the plasmids could self-transmission, we used the oriTfinder ${ }^{4}$ to conduct a detailed analysis of the conjugation module, including the origin of transfer site (oriT), relaxase gene, type IV coupling protein (T4CP) gene, and the type IV secretion system gene cluster (T4SS). The related insertion sequences (IS)

\footnotetext{
${ }^{1}$ https://github.com/ruanjue/smartdenovo

${ }^{2}$ https://cge.cbs.dtu.dk/services/PlasmidFinder/

${ }^{3}$ https://cge.cbs.dtu.dk/services/ResFinder/

${ }^{4}$ https://tool-mml.sjtu.edu.cn/oriTfinder/oriTfinder.html
}

and transposons (Tn) were determined through the ISFinder ${ }^{5}$. BLAST Ring Image Generator (BRIG) was used to compare key resistant plasmids with other representative plasmids to further generate circular plasmid maps. Easyfig software was used to generate comparison of gene environment surrounding the vital resistant genes.

\section{Transformation Assay}

In order to test whether these plasmids could mediate the corresponding resistant phenotype, we extracted and transformed each single resistant plasmid to E. coli Top 10, and then tested the antibiotic susceptibility of all transformants. The plasmid extraction and transformation processes were performed as previously described (Yin et al., 2017).

We used the phenol-chloroform extraction method to extract the plasmids in 1678. Then, we mixed $4 \mu$ l extracted plasmids and E. coli Top 10 competent cells together, placed it on ice for $30 \mathrm{~min}$, put it in a $42^{\circ} \mathrm{C}$ water bath for $90 \mathrm{~s}$, and then took it out and placed it on ice for $2 \mathrm{~min}$. After that, we used LB broth to resuscitate the strain, and screened the transformants on appropriate antibiotic plates. Successful transformants were determined by PCR. All the transformants were selected in appropriate antibiotics [Amp, $100 \mathrm{mg} / \mathrm{L}$ (bla $a_{\mathrm{NDM}-5}$ ); Fosfomycin, $16 \mathrm{mg} / \mathrm{L}$ (fosA3); colistin, $4 \mathrm{mg} / \mathrm{L}$ ( $m c r-1)$; tetracycline, $30 \mathrm{mg} / \mathrm{L}($ tet $A)]$.

\section{Conjugation Assay}

We applied conjugation assay (Zhou et al., 2020) to evaluate whether these resistant plasmids could be transferred or cotransferred from K. pneumoniae 1678 (donor isolate) to E. coli J53 (recipient isolate). The donors and recipients were cultured to the logarithmic phase, mixed in 1:1 ratio, centrifuged at $8,000 \times g$ for $1 \mathrm{~min}$, and then resuspended in $20 \mu \mathrm{l} \mathrm{MgSO}_{4}$ $(10 \mathrm{mM})$. The resuspension was spotted on the Luria Bertani (LB) plate and incubated at $37^{\circ} \mathrm{C}$ overnight. Subsequently, the serial dilutions were plated in media with appropriate antibiotics [Amp, $100 \mathrm{mg} / \mathrm{L}\left(b l a_{\mathrm{NDM}-5}\right)$; Fosfomycin, $16 \mathrm{mg} / \mathrm{L}$ (fosA3); colistin, $4 \mathrm{mg} / \mathrm{L}$ ( $m c \mathrm{r}-1$ ); sodium azide, $100 \mathrm{mg} / \mathrm{L}$ (J53 recipient)]. The conjugation frequency was calculated as the number of transconjugants per donor. All transconjugants were confirmed by PCR for the presence of $f o s A 3, b l a_{\mathrm{NDM}-5}$, and $m c r-1$ genes. All the primers were listed in Supplementary Table 1.

\section{S1-Pulsed-Field Gel Electrophoresis Assay}

The S1-pulsed-field gel electrophoresis (S1-PFGE) was performed to further determine the existence of plasmids in the original isolate K. pneumoniae 1678, and its transformants and transconjugants. PFGE plugs of all these strains were prepared and digested as previously described (Ai et al., 2021). Briefly, the isolates were embedded in $10 \mathrm{~g} / \mathrm{L}$ of Seakem Gold gel, and digested with endonuclease S1 nuclease (Takara, Dalian, China). PFGE analysis was performed with a CHEF mapper system (Bio-Rad). The digested DNA fragments were separated for $19 \mathrm{~h}$ at $6 \mathrm{~V} / \mathrm{cm}, 14^{\circ} \mathrm{C}$ on a $1.0 \%$ agarose gel (Bio-Rad) with

${ }^{5}$ https://www-is.biotoul.fr/ 
pulse times of 4-40 s. XbaI-digested Salmonella H9812 DNA was used as the DNA marker. The nucleic acid dye Gel-red (Yeasen, China) was used to stain the DNA in the gels.

\section{Nucleotide Sequence Accession Numbers of Klebsiella pneumoniae 1678}

The complete nucleotide sequences of the chromosome and plasmids p1678-2, p1678-3, p1678-4, p1678-5, and p1876 were submitted to GenBank under accession numbers CP080445, CP080446, CP080447, CP080448, CP080449, and CP080450, respectively.

\section{RESULTS}

\section{Klebsiella pneumoniae 1678 Was a Typical Extensively Drug-Resistant Isolate}

In order to clarify the antibiotic-resistant phenotype of Klebsiella pneumoniae 1678, we tested the susceptibility of 26 antibiotics in this strain (Table 1), especially including Fosfomycin, tigecycline, colistin, and ceftazidime-avibactam that were known for their robust bactericidal effect against CRKP. Our results indicated the K. pneumoniae 1678 was a representative multi-drug resistant strain, which not only exhibited high-level resistance to all $\beta$-lactam antibiotics and carbapenems, but was even resistant to tigecycline, Fosfomycin, colistin, and ceftazidime-avibactam (Table 1). These resistance profiles indicated the treatment option for the infection caused by K. pneumoniae 1678 would be limited.

\section{Klebsiella pneumoniae 1678 Co-harboring fos $A 3$, bla NDM -5 , and $m c r-1$}

To further investigate the related mechanism that mediated the extensively drug-resistant resistant (XDR) characteristic of K. pneumoniae 1678, we used WGS to deeply describe the genomic information of the XDR bacteria. According to the MLST analysis, the K. pneumoniae 1678 was typed as ST485. We found more than 20 resistant elements and 5 resistant plasmids in this isolate (Table 2). Moreover, three key resistance genes were focused, which played a significant role in the formation of resistance to Fosfomycin (fosA3), carbapenems, ceftazidimeavibactam $\left(b l a_{\mathrm{NDM}-5}\right)$, and colistin $(m c r-1)$. In addition to the molecular detection of these crucial resistant elements, we also extracted and transformed each resistant plasmid to E. coli Top 10 (Figure 1A) and tested whether these plasmids could mediate the corresponding resistant phenotype. Antibiotic susceptibility results of all transformants well proved the role of the resistant plasmids (Table 2).

\section{Tigecycline Resistance Was Mediated by the Overexpression of RND-Type Efflux Transporters}

The mechanisms underlying tigecycline resistance are complex. Previous studies demonstrated the mutation of plasmid-borne tet $(A)$ could be an important factor causing tigecycline resistance in K. pneumoniae. Accordingly, we compared the amino-acid sequence of the Tet(A) protein of K. pneumoniae 1678 and the mutated Tet(A) confirmed before (Supplementary Figure 1). We found the Tet(A) carried by K. pneumoniae 1678 owned the same mutated characteristic which $\mathrm{Xu}$ (Xu et al., 2021) described (Supplementary Figures 1, 3). Moreover, we also detected the mRNA expression of the tet $(A)$ gene in the $E$. coli transformants to ensure the tet( $A$ ) gene could be expressed normally (Supplementary Figure 2). However, we could not detect the tigecycline resistant phenotype, but only the resistant to tetracycline in the tet(A)-transformants (Table 2), which indicated that this mutation may not contribute to tigecycline resistance in K. pneumoniae 1678.

In addition to the mutation of $\operatorname{Tet}(\mathrm{A})$ protein, the overexpression of the RND-type efflux pumps AcrAB and OqxAB has been shown to play a crucial role in tigecycline resistance in K. pneumoniae (Bialek-Davenet et al., 2015; Li et al., 2017). Our qRT-PCR experiments indicated that K. pneumoniae 1678 overexpressed the AcrAB-TolC pathway genes $\operatorname{acr} A / B$ and tolC (>sixfold greater than the tigecycline susceptible K. pneumoniae ATCC 13883 reference strain) (Figure 2A), and was also observed to overexpress oqxA and oq $x B$ (range 6.432 - to 10.435 -fold compared with the reference strain levels) (Figure 2B). Moreover, the activating regulator of AcrAB $(\operatorname{ramA})$ and OqxAB (rarA) also exhibited the same expression level (Figure 2). What is more, the mutation in RamR protein was also analyzed, for it is the negative regulator of RamA (Zheng et al., 2018), and we found an amino acid mutation (L44M) compared with the reference sequence (Accension number: ADI49705.1), but the phenotype is unproved. We assumed this may do a potential favor for the overexpression of AcrAB. These results showed the overexpression of RND-type efflux transporters contributed to the tigecycline resistance of K. pneumoniae 1678.

\section{Comparative Genomics of the Plasmids Carrying Resistance Genes}

We have confirmed that the key resistant genes were all located on plasmids. As plasmids are often transmissible between bacteria, and some have spread globally, we made detailed analysis of these resistant plasmids, aiming to further clarify the resistance mechanism and potential dissemination threats of $K$. pneumoniae 1678. p1678-3 was a typical IncFII-type plasmid, harboring a completed conjugation system, and shared $81 \%$ identity with pFOS-HK151325, the first fos 33 plasmid from a clinical E. coli identified in China (Figure 3A). Moreover, p1678-3 was also highly similar to pKP32558-4 (89\% identity, CP076034.1, K. pneumoniae) and p116753-KPC (95\% identity, MN891682.1, $K$. pneumoniae). The genetic differences between p1678-3 and these plasmids were most concentrated in the surrounding genes of fos $A 3$ gene, which may be related to the mobility insertion of IS elements (Figure 3A).

The wide dissemination of $b l a_{\mathrm{NDM}}$ genes is largely mediated by certain plasmids, particularly those of the IncX3 type, which p1678-4 plasmid belonged to. Moreover, the genetic context of the p1678-4 plasmid (Figure 4A) was nearly identical to that of the human K. pneumoniae plasmid pNDM-MGR194 (2015, IncX3, bla $\left.\mathrm{NDM}_{-5}, \mathrm{KF} 220657.1\right)$ previously reported in India (Krishnaraju et al., 2015) and was also highly similar to p2B8067 
TABLE 1 | Antimicrobial drug susceptibility profiles.

\begin{tabular}{|c|c|c|c|c|c|c|c|c|c|c|c|c|c|}
\hline \multirow[t]{3}{*}{ Antibiotics } & \multicolumn{13}{|c|}{ MIC (mg/L)/antimicrobial susceptibility } \\
\hline & \multirow[b]{2}{*}{1678} & \multirow[b]{2}{*}{ Top10 } & \multirow[b]{2}{*}{ J53 } & \multicolumn{4}{|c|}{ Transformants } & \multicolumn{6}{|c|}{ Transconjugants } \\
\hline & & & & $\begin{array}{l}\text { p1678-6- } \\
\text { TOP10 } \\
\text { (TetA) }\end{array}$ & $\begin{array}{c}\text { p1678-3 } \\
\text { Top10 } \\
\text { (FosA3) }\end{array}$ & $\begin{array}{c}\text { p1678-4- } \\
\text { Top10 } \\
\text { (NDM-5) }\end{array}$ & $\begin{array}{l}\text { p1678-5-Top10 } \\
\text { (MCR-1) }\end{array}$ & $\begin{array}{l}\text { p1678-3-J53 } \\
\text { (FosA3) }\end{array}$ & $\begin{array}{l}\text { p1678-4-J53 } \\
\text { (NDM-5) }\end{array}$ & $\begin{array}{l}\text { p1678-5-J53 } \\
\text { (MCR-1) }\end{array}$ & $\begin{array}{l}\text { p1678-3 and 5-J53 } \\
\text { (FosA3+MCR-1) }\end{array}$ & $\begin{array}{l}\text { p1678-4 and } \\
5-J 53 \\
\text { (NDM- } \\
\text { 5+MCR-1) }\end{array}$ & $\begin{array}{c}\mathrm{p} 1678-3 \text { and } 4 \text { and } \\
5-\mathrm{J} 53 \\
\text { (FosA3+NDM- } \\
\mathbf{5 + M C R - 1 )}\end{array}$ \\
\hline MEM & $>16 / R$ & $\leq 0.06 / \mathrm{S}$ & $\leq 0.06 / \mathrm{S}$ & $\leq 0.06 / \mathrm{S}$ & $\leq 0.06 / S$ & $16 / R$ & $\leq 0.06 / \mathrm{S}$ & $\leq 0.06 / \mathrm{S}$ & $16 / R$ & $\leq 0.06 / \mathrm{S}$ & $\leq 0.06 / \mathrm{S}$ & $16 / R$ & $16 / R$ \\
\hline IPM & $16 / R$ & $\leq 0.25 / \mathrm{S}$ & $\leq 0.25 / \mathrm{S}$ & $\leq 0.25 / S$ & $\leq 0.25 / \mathrm{S}$ & $4 / R$ & $\leq 0.25 / \mathrm{S}$ & $\leq 0.25 / \mathrm{S}$ & 4/R & $\leq 0.25 / \mathrm{S}$ & $\leq 0.25 / \mathrm{S}$ & 8/R & $8 / R$ \\
\hline ETP & $>2 / R$ & $\leq 0.015 / \mathrm{S}$ & $\leq 0.015 / \mathrm{S}$ & $\leq 0.015 / \mathrm{S}$ & $\leq 0.015 / \mathrm{S}$ & $>2 / R$ & $\leq 0.015 / \mathrm{S}$ & $\leq 0.015 / \mathrm{S}$ & $>2 / R$ & $\leq 0.015 / \mathrm{S}$ & $\leq 0.015 / \mathrm{S}$ & $>2 / R$ & $>2 / R$ \\
\hline Caz/AVI & $>16 / 4 / R$ & $\leq 0.5 / 4 / S$ & $\leq 0.5 / 4 / \mathrm{S}$ & $\leq 0.5 / 4 / S$ & $\leq 0.5 / 4 / \mathrm{S}$ & $>16 / 4 / R$ & $\leq 0.5 / 4 / \mathrm{S}$ & $\leq 0.5 / 4 / \mathrm{S}$ & $>16 / 4 / R$ & $\leq 0.5 / 4 / \mathrm{S}$ & $\leq 0.5 / 4 / \mathrm{S}$ & $>16 / 4 / R$ & $>16 / 4 / R$ \\
\hline TGC & $8 / R$ & $\leq 0.25 / \mathrm{S}$ & $\leq 0.25 / \mathrm{S}$ & $\leq 0.25 / \mathrm{S}$ & $\leq 0.25 / \mathrm{S}$ & $\leq 0.25 / \mathrm{S}$ & $\leq 0.25 / \mathrm{S}$ & $\leq 0.25 / \mathrm{S}$ & $\leq 0.25 / \mathrm{S}$ & $\leq 0.25 / \mathrm{S}$ & $\leq 0.25 / \mathrm{S}$ & $\leq 0.25 / \mathrm{S}$ & $\leq 0.25 / \mathrm{S}$ \\
\hline POL & $16 / R$ & $0.25 / \mathrm{S}$ & $0.25 / \mathrm{S}$ & $0.25 / \mathrm{S}$ & $0.25 / \mathrm{S}$ & $0.25 / \mathrm{S}$ & $16 / R$ & $0.25 / \mathrm{S}$ & $0.25 / \mathrm{S}$ & $16 / R$ & $16 / R$ & $16 / R$ & $16 / R$ \\
\hline FOS & $>256 / R$ & $0.25 / \mathrm{S}$ & $0.25 / \mathrm{S}$ & $0.25 / \mathrm{S}$ & $>256 / R$ & $0.25 / \mathrm{S}$ & $0.25 / \mathrm{S}$ & $>256 / R$ & $0.25 / \mathrm{S}$ & $0.25 / \mathrm{S}$ & $>256 / R$ & $0.25 / \mathrm{S}$ & $>256 / R$ \\
\hline AMP & $>32 / R$ & $\leq 8 / S$ & $\leq 8 / S$ & $\leq 8 / S$ & $>32 / R$ & $>32 / R$ & $\leq 8 / S$ & $>32 / R$ & $>32 / \mathrm{R}$ & $\leq 8 / S$ & $>32 / R$ & $>32 / R$ & $>32 / R$ \\
\hline CZO & $>32 / \mathrm{R}$ & $\leq 2 / S$ & $\leq 2 / S$ & $\leq 2 / S$ & $>32 / R$ & $>32 / \mathrm{R}$ & $\leq 2 / S$ & $>32 / R$ & $>32 / \mathrm{R}$ & $\leq 2 / S$ & $>32 / \mathrm{R}$ & $>32 / R$ & $>32 / \mathrm{R}$ \\
\hline CAZ & $>128 / R$ & $\leq 0.25 / \mathrm{S}$ & $\leq 0.25 / \mathrm{S}$ & $\leq 0.25 / S$ & $>128 / R$ & $>128 / R$ & $\leq 0.25 / \mathrm{S}$ & $>128 / R$ & $>128 / R$ & $\leq 0.25 / \mathrm{S}$ & $>128 / R$ & $>128 / R$ & $>128 / R$ \\
\hline FEP & $>16 / R$ & $\leq 0.5 / S$ & $\leq 0.5 / S$ & $\leq 0.5 / S$ & $\leq 0.5 / \mathrm{S}$ & $>16 / R$ & $\leq 0.5 / S$ & $\leq 0.5 / \mathrm{S}$ & $>16 / R$ & $\leq 0.5 / S$ & $\leq 0.5 / S$ & $>16 / R$ & $>16 / R$ \\
\hline CSL & $>64 / 32 / R$ & $\leq 16 / 8 / S$ & $\leq 16 / 8 / \mathrm{S}$ & $\leq 16 / 8 / S$ & $\leq 16 / 8 / S$ & $>64 / 32 / \mathrm{R}$ & $\leq 16 / 8 / \mathrm{S}$ & $\leq 16 / 8 / \mathrm{S}$ & $>64 / 32 / R$ & $\leq 16 / 8 / S$ & $\leq 16 / 8 / S$ & $>64 / 32 / R$ & $>64 / 32 / R$ \\
\hline SAM & $>32 / 16 / R$ & $\leq 16 / 4 / S$ & $\leq 16 / 4 / \mathrm{S}$ & $\leq 16 / 4 / S$ & $\leq 16 / 4 / S$ & $>32 / 16 / R$ & $\leq 16 / 4 / S$ & $\leq 16 / 4 / \mathrm{S}$ & $>32 / 16 / R$ & $\leq 16 / 4 / S$ & $\leq 16 / 4 / \mathrm{S}$ & $>32 / 16 / R$ & $>32 / 16 / R$ \\
\hline FOX & $>32 / R$ & $\leq 8 / S$ & $\leq 8 / S$ & $\leq 8 / S$ & $\leq 8 / S$ & $>32 / R$ & $\leq 8 / S$ & $\leq 8 / S$ & $>32 / R$ & $\leq 8 / S$ & $\leq 8 / \mathrm{S}$ & $>32 / \mathrm{R}$ & $>32 / R$ \\
\hline CXM & $>16 / R$ & $8 / 5$ & $8 / S$ & $8 / \mathrm{S}$ & $>16 / R$ & $>16 / R$ & $8 / \mathrm{S}$ & $>16 / R$ & $>16 / R$ & $8 / S$ & $>16 / R$ & $>16 / R$ & $>16 / R$ \\
\hline CTX & $>64 / R$ & $\leq 0.12 / \mathrm{S}$ & $\leq 0.12 / S$ & $\leq 0.12 / S$ & $64 / R$ & $>64 / R$ & $\leq 0.12 / S$ & $64 / R$ & $>64 / R$ & $\leq 0.12 / S$ & $64 / R$ & $>64 / R$ & $>64 / R$ \\
\hline TZP & $>128 / 4 / R$ & $\leq 16 / 4 / \mathrm{S}$ & $\leq 16 / 4 / S$ & $\leq 16 / 4 / S$ & $\leq 16 / 4 / \mathrm{S}$ & $>128 / 4 / R$ & $\leq 16 / 4 / S$ & $\leq 16 / 4 / S$ & $>128 / 4 / R$ & $\leq 16 / 4 / S$ & $\leq 16 / 4 / S$ & $>128 / 4 / R$ & $>128 / 4 / R$ \\
\hline AMC & $>32 / 16 / R$ & $\leq 8 / 4 / S$ & $\leq 8 / 4 / S$ & $\leq 8 / 4 / S$ & $\leq 8 / 4 / S$ & $>32 / 16 / R$ & $\leq 8 / 4 / S$ & $\leq 8 / 4 / S$ & $>32 / 16 / \mathrm{R}$ & $\leq 8 / 4 / S$ & $\leq 8 / 4 / S$ & $>32 / 16 / R$ & $>32 / 16 / \mathrm{R}$ \\
\hline LVX & $>8 / R$ & $\leq 0.12 / \mathrm{S}$ & $\leq 0.12 / \mathrm{S}$ & $\leq 0.12 / \mathrm{S}$ & $\leq 0.12 / \mathrm{S}$ & $\leq 0.12 / \mathrm{S}$ & $\leq 0.12 / \mathrm{S}$ & $\leq 0.12 / \mathrm{S}$ & $\leq 0.12 / \mathrm{S}$ & $\leq 0.12 / \mathrm{S}$ & $\leq 0.12 / \mathrm{S}$ & $\leq 0.12 / \mathrm{S}$ & $\leq 0.12 / \mathrm{S}$ \\
\hline MFX & $>2 / R$ & $\leq 0.25 / \mathrm{S}$ & $\leq 0.25 / \mathrm{S}$ & $\leq 0.25 / \mathrm{S}$ & $\leq 0.25 / \mathrm{S}$ & $\leq 0.25 / \mathrm{S}$ & $\leq 0.25 / \mathrm{S}$ & $\leq 0.25 / \mathrm{S}$ & $\leq 0.25 / \mathrm{S}$ & $\leq 0.25 / \mathrm{S}$ & $\leq 0.25 / \mathrm{S}$ & $\leq 0.25 / \mathrm{S}$ & $\leq 0.25 / \mathrm{S}$ \\
\hline TCY & $>16 / R$ & $\leq 2 / S$ & $\leq 2 / S$ & $16 / R$ & $\leq 2 / S$ & $\leq 2 / S$ & $\leq 2 / S$ & $\leq 2 / S$ & $\leq 2 / S$ & $\leq 2 / S$ & $\leq 2 / S$ & $\leq 2 / S$ & $\leq 2 / S$ \\
\hline GEN & $16 / R$ & $\leq 1 / S$ & $\leq 1 / S$ & $\leq 1 / S$ & $\leq 1 / S$ & $\leq 1 / S$ & $\leq 1 / S$ & $\leq 1 / S$ & $\leq 1 / S$ & $\leq 1 / S$ & $\leq 1 / S$ & $\leq 1 / S$ & $\leq 1 / S$ \\
\hline AMK & $\leq 16 / \mathrm{S}$ & $\leq 16 / S$ & $\leq 16 / S$ & $\leq 16 / S$ & $\leq 16 / \mathrm{S}$ & $\leq 16 / S$ & $\leq 16 / S$ & $\leq 16 / S$ & $\leq 16 / S$ & $\leq 16 / S$ & $\leq 16 / S$ & $\leq 16 / S$ & $\leq 16 / S$ \\
\hline ATM & $>16 / R$ & $\leq 4 / S$ & $\leq 4 / S$ & $\leq 4 / S$ & $>16 / R$ & $\leq 4 / S$ & $\leq 4 / S$ & $>16 / R$ & $\leq 4 / S$ & $\leq 4 / S$ & $\leq 4 / S$ & $\leq 4 / S$ & $>16 / R$ \\
\hline NIT & $64 / 1$ & $\leq 16 / \mathrm{S}$ & $\leq 16 / \mathrm{S}$ & $\leq 16 / \mathrm{S}$ & $\leq 16 / S$ & $\leq 16 / \mathrm{S}$ & $\leq 16 / \mathrm{S}$ & $\leq 16 / S$ & $\leq 16 / \mathrm{S}$ & $\leq 16 / \mathrm{S}$ & $\leq 16 / \mathrm{S}$ & $\leq 16 / S$ & $\leq 16 / \mathrm{S}$ \\
\hline SXT & $>4 / 76 / R$ & $\leq 0.5 / 9.5 / \mathrm{S}$ & $\leq 0.5 / 9.5 / \mathrm{S}$ & $\leq 0.5 / 9.5 / \mathrm{S}$ & $\leq 0.5 / 9.5 / \mathrm{S}$ & $\leq 0.5 / 9.5 / \mathrm{S}$ & $\leq 0.5 / 9.5 / \mathrm{S}$ & $\leq 0.5 / 9.5 / \mathrm{S}$ & $\leq 0.5 / 9.5 / \mathrm{S}$ & $\leq 0.5 / 9.5 / \mathrm{S}$ & $\leq 0.5 / 9.5 / \mathrm{S}$ & $\leq 0.5 / 9.5 / \mathrm{S}$ & $\leq 0.5 / 9.5 / \mathrm{S}$ \\
\hline
\end{tabular}

MEM, Meropenem; IPM, Imipenem; ETP, Ertapenem; Caz/AVI, ceftazidime-avibactam; TGC, Tigecycline; POL, Polymixin B; FOS, Fosfomycin; AMP, Ampicillin; CZO, Cefazolin; CAZ, Ceftazidime; FEP, Cefepime; CSL, Cefoperazone/Sulbactam; SAM, Ampicillin/Sulbactam; FOX, Cefoxitin; CXM, Cefuroxime; CTX, Cefotaxime; TZP, Piperacillin/Tazobactam; AMC, Amoxicillin/Clavulanic acid; LVX, Levofloxacin; MFX, Moxifloxacin; TCY, Tetracycline; GEN, Gentamicin; AMK, Amikacin; ATM, Aztreonam; NIT, Nitrofurantoin; SXT, Trimethoprim/Sulfamethoxazole.

The other resistance phenotype like LVX, MFX, GEN. NIT, or SXT resistance did not present in these transformants or transconjugants in table because these elements are located on p1678-2 plasmid, not p16783-

3(fosA3), p1678-4(bla NDM-5), p1678-5(mcr-1), and p1678-6(tetA). The bold values indicated important resistance genes and resistance phenotypes. 
TABLE 2 | General features, antimicrobial resistance genes of plasmids in K. pneumoniae 1678 .

\begin{tabular}{|c|c|c|c|c|c|}
\hline \multirow[t]{2}{*}{ Characteristics } & \multicolumn{5}{|c|}{ Results } \\
\hline & p1678-2 & p1678-3 & p1678-4 & p1678-5 & p1678-6 \\
\hline Accension number & СР080446 & CP080447 & CP080448 & CP080449 & СР080450 \\
\hline Length(bp) & 90,943 & 76,526 & 46,161 & 33,309 & 24,774 \\
\hline GC content (\%) & 54 & 52 & 47 & 42 & 54 \\
\hline No. of ORF & 116 & 92 & 59 & 42 & 29 \\
\hline \multicolumn{2}{|c|}{ Incompatibility group IncFIIK(IncQ1 } & IncFII & $\operatorname{lncX} 3$ & IncX4 & IncR \\
\hline Conjugal ability & No & Yes & Yes & Yes & No \\
\hline \multicolumn{6}{|l|}{ Resistant genes } \\
\hline & bla oXA-1 & fosA3 & $b_{\text {laDM-5 }}$ & $m c r-1$ & TetA \\
\hline & aac(3)-lid & bla $\mathrm{CTX}-\mathrm{M}-55$ & & & \\
\hline & aph(3')-la & blaTEM-141 & & & \\
\hline & sul2, sul1 & & & & \\
\hline & sul2, sul1; & & & & \\
\hline & $\mathrm{aac}\left(6^{\prime}\right)-\mathrm{lb}-\mathrm{cr}$ & & & & \\
\hline & aph(3')-la & & & & \\
\hline & aac(3)-lid & & & & \\
\hline & $\operatorname{mph}(A)$ & & & & \\
\hline & aadA16 & & & & \\
\hline & qnrB52 & & & & \\
\hline & ARR-3 & & & & \\
\hline & catB3 & & & & \\
\hline
\end{tabular}

The bold values indicated important resistance genes and resistance phenotypes.

(2021, IncX3, bla $\left.a_{\mathrm{NDM}-7}, \mathrm{CP} 070442.1\right)$. These results indicated that no matter the variants of $b l a_{\mathrm{NDM}}$, the IncX3 plasmid was a major vehicle in mediating the dissemination of $b l a_{\mathrm{NDM}}$. Similar to the IncFII plasmids described before (Bi et al., 2018), IncX3 plasmid also could be self-transferred among Enterobacteriaceae, supported with the results of the conjugation mode analysis of p1678-4 plasmid (Table 2).

p1678-5 plasmid was a 33,309-bp circular molecule with repA belonging to IncX 4 , harboring $m c r-1$ resistance element (Figure 5A). Previous studies have demonstrated most plasmids carrying $m c r-1$ are transferable, and IncX4 was dominant $m c r-1$ carrying plasmid types (Xiaomin et al., 2020). According to the genomic comparation, we found p1678-5 was almost identical to both pQDFD216-1 (CP053212.1) plasmid identified in E. coli and plasmid 16BU137 (MT316509.1) from K. pneumoniae. These results showed IncX4 plasmids harboring $m c r-1$ could disseminate in different species of Enterobacteriaceae, and the completed conjugative element also can be found in p16785 plasmid (Table 2). In addition, we also make a comparison between p1678-5 and the first mcr-1 plasmid pHNSHP45 (NZ_KP347127.1, IncI2) (Liu et al., 2016), and found low identity between two plasmids (Supplementary Figure 4).

\section{Resistant Plasmids Could Be High-Efficient Self-Transferred or Co-transferred}

We have known these three resistance plasmids were all bioinformatic predicted to carry essential conjugative modules
(oriT, Relaxase, T4CP, and T4SS) and most of these types of plasmids have proved to be movable (Partridge et al., 2018). However, it is not common for such three plasmids to co-exist in one K. pneumoniae, and the transferring and co-transferring pattern was unclear. Here, we applied conjugation assay to imitate and evaluate the dissemination ability of these three plasmids in $K$. pneumoniae 1678 . We found all these three plasmids could transfer to $E$. coli J53 with high conjugation frequencies $\left(1.42 \times 10^{-4}-7.9 \times 10^{-3}\right)$, especially for the mcr-1 plasmid (p1678-5) (Table 3 ). In addition to the selftransferring of a single plasmid, we also observed co-transfer of two plasmids and even three plasmids and the co-conjugation frequencies of two plasmids only decrease 1-log compared to a single plasmid (Table 3). Although the co-conjugation frequencies of three plasmids was low, the potential clinical threat could not be ignored, since the clonal spread will accelerate the spread of these resistance genes. Moreover, the plasmid pattern of S1-PFGE further proved the transferring profile of the K. pneumoniae 1678 (Figure 1B), and the antibiotic MICs of these transconjugants also confirmed the spread of resistance phenotype of K. pneumoniae 1678 (Table 1).

\section{Mobile Genetic Elements Associated With fosA3, bla $\mathrm{NDM}-5$, and $m c r-1$}

The capture, accumulation, and dissemination of resistance genes are not only due to the spread of plasmids, but also to the actions of other MGEs, such as IS and Tn. To comprehensively evaluate the dissemination potential of these resistance genes in $K$. pneumoniae 1678, we also analyze the MGEs surrounding them. IS26 seems to be the key element in the mobilization of fos $A 3$, since it not only composes a composite transposon surrounding fos $A 3$ in the p1678-3 plasmids, but also surrounding the fosA3 of pFOS-HK151325 plasmid (Yang et al., 2019) (2013, JX627737, E. coli, China), of pFOS18 plasmid (Yang et al., 2019) (2015, KJ653815, K. pneumoniae, China), and of p06607 plasmid (Yang et al., 2019) (2010, AB522970, first fosA3 plasmid emerged in the world). Moreover, the same IS26-composite transposons were also observed to frequently contain additionally a bla $a_{\mathrm{CTX}-\mathrm{M}}$ gene. Compared to the first fos $A 3$ plasmid, there are more than 10 ORFs inserted around fos $A 3$ in p1678-3 plasmid, with some new IS, some elements associated with transcriptional regulation, and other resistance elements (Figure 3B).

The genetic contexts of bla $a_{\mathrm{NDM}}$ share two common features. The insertion sequence ISAba125 (intact or truncated) is always upstream of $b l a_{\mathrm{NDM}}$, while a bleomycin resistance gene, ble $e_{\mathrm{MBL}}$, is always downstream. Further downstream of ble $e_{\mathrm{MBL}}$, there are usually located $\operatorname{trp} F$ and $d s b C$ genes (Figure 4B). Although the bla $a_{\mathrm{NDM}}-1$ in Acinetobacter spp. is located within ISAba125based composite transposon Tn125(pNDM-BJ01, JQ001791) (Wu et al., 2019), it was always interrupted or truncated in Enterobacteriaceae. In p1678-4, Tn 125 was truncated by IS5, IS26, and IS300 (Figure 4B). These new genetic contexts in p16784 may form a new mechanism involved in the mobilization of bla $a_{\mathrm{NDM}-5}$.

Similar to other mcr-1-carrying IncX4-type plasmids (Xiaomin et al., 2020), the typical IS26-parA-mcr-1.1-pap2 
A

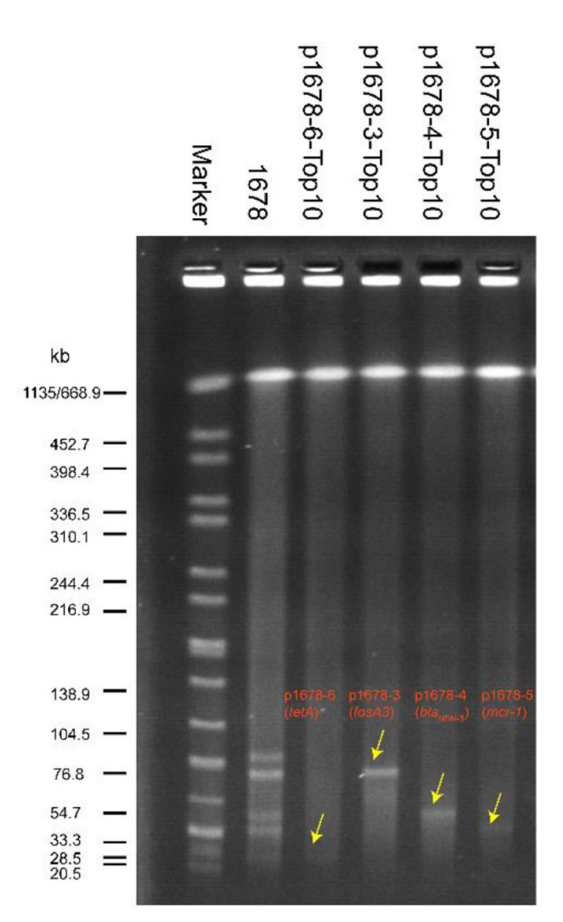

B

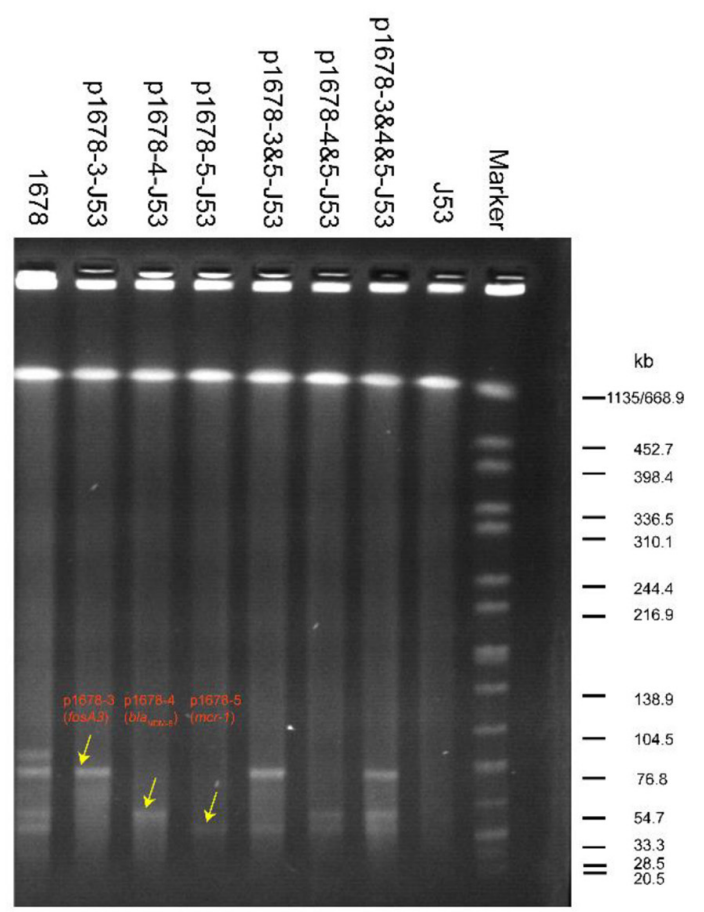

FIGURE 1 | SI-PFGE profiles of original K. pneumoniae 1678 and its transformants (A) and transconjugants (B). Lane marker was Xbal-digested DNA of Salmonella Braenderup H9812; Lane 1678 and Lane J53 were used as positive reference and negative control, respectively; transformants: p1678-6-Top10 (tetA), p1678-3-Top10 (fosA3), p1678-4-Top10(bla NDM-5), and p1678-5-Top10(mcr-1). Transconjugants: p1678-3-J53(fosA3), p1678-4-J53(bla NDM-5), p1678-5-J53(mcr-1), p1678-3 and 5-J53 (fosA3 and mcr-1), p1678-4 and 5-J53 (blandM-5 and mcr-1), and p1678-3 and 4 and 5-J53 (fosA3, blandM-5, and mcr-1).

\section{A}

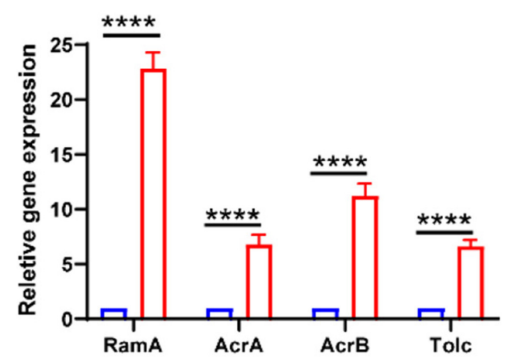

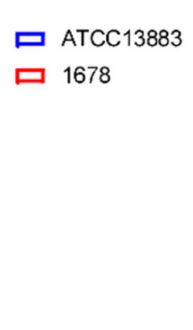

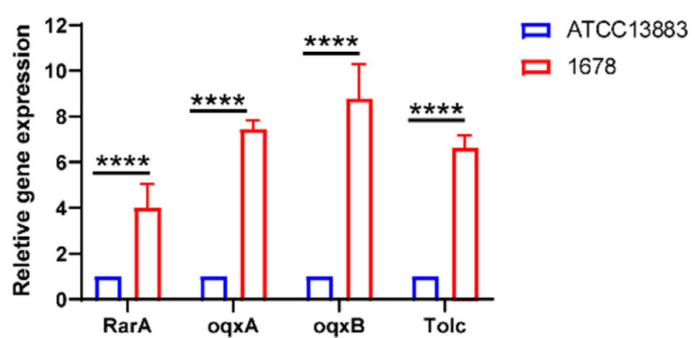

FIGURE 2 | Overexpression of AcrAB and OqxAB in clinical K. pneumoniae 1678 isolate. The expression levels of AcrA/B (A), OqxA/B (B), and related transcriptional regulators was determined by qRT-PCR. The $K$. pneumoniae ATCC13883 is used as the reference strain (expression = 1.0). The data represent the mean standard deviation for three independent biological replicates. Differences between different strains, regarding related gene expression, were statistically analyzed using a two-tailed Student's $t$-test with Bonferroni correction. ${ }^{\star * \star *} p<0.0001$.

cassette was identified in p1678-5, with pap2 gene partitional truncated (Figure 5B). Although ISApl1 has been described as the most common IS element adjacent to $m c r-1$ at one or both ends, we did not observe it surrounding the $m \mathrm{mor}-1$ gene.

\section{DISCUSSION}

Extensively drug-resistant $K$. pneumoniae constitutes the major sources of nosocomial infections with extraordinary drug resistance. The prevalent resistance plasmids are responsible for the sudden increase in the population of multidrug resistance among $K$. pneumoniae isolates (Tzouvelekis et al., 2012; Chen et al., 2014). In this study, we described a multi-drug resistant $K$. pneumoniae 1678 co-harboring three self-transmissible resistant plasmids, which mediated the resistance for carbapenems, Fosfomycin, colistin, and ceftazidime-avibactam. The co-existing of these plasmids not only conferred the multi-drug resistant phenotype to $K$. pneumoniae 


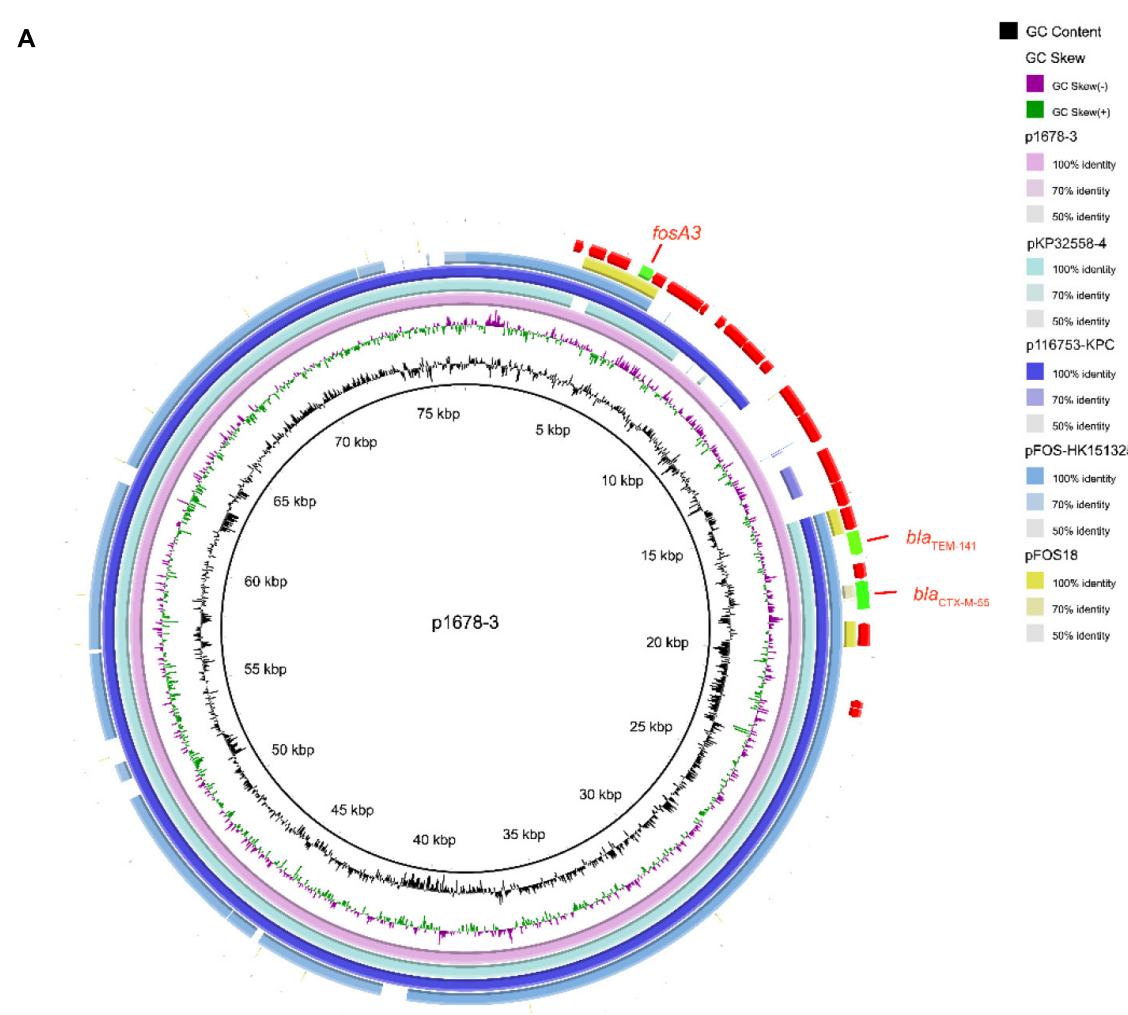

B

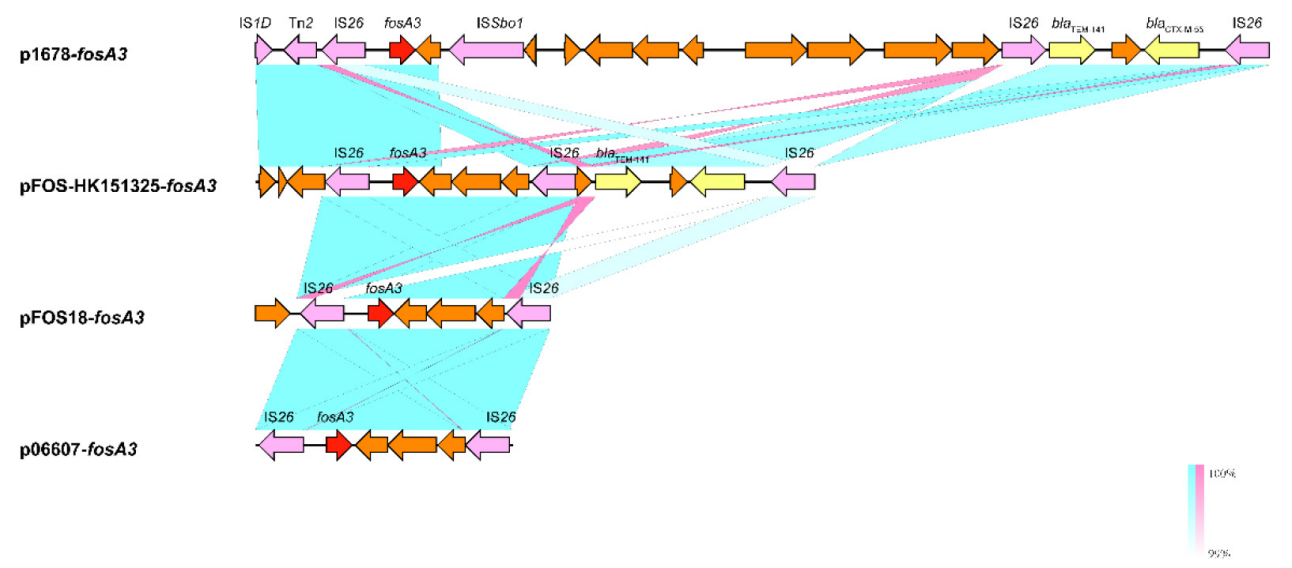

FIGURE 3 | Comparative analysis of pl687-3 plasmids with other reference plasmids. (A) p1678-3 (CP080447) was used as the reference plasmid to perform genome alignment with pFOS-HK151325 (JX627737, first fosA3 plasmid from a clinical E. coli identified in China) and pFOS18 (KJ653815, first fosA3 plasmid from a clinical K. pneumoniae identified in China). Moreover, p1678-3 was also compared with another two similar plasmids pKP32558-4 (CP076034.1, K. pneumoniae) and p116753-KPC (MN891682.1, K. pneumoniae). The red arrows represent CDs. (B) Linear comparison of the fosA3 region. The fosA3 region was compared with the regions extracted from pFOS-HK151325, pFOS18, and p06607 (AB522970, first fosA3 plasmid).

1678, but also held the potential threat to co-transfer to other isolates.

Tigecycline has been considered as an effective antibiotic against CRKP in vitro, and is also considered as one of the lastresort antibiotics against CRKP infections (Chopra, 2002; Doi, 2019). Unfortunately, tigecycline resistance has been reported frequently in clinics (Fang et al., 2020). In this study, we found
K. pneumoniae 1678 was resistant to tigecycline. We identified the $\operatorname{tet}(A)$ gene in this isolate, and also confirmed the mutations on Tet(A) protein (Supplementary Figure 1). Several studies had verified the plasmid-borne mutated tet $(A)$ gene or the synergy of TetA and RND-type efflux transporters play an important role in causing tigecycline resistance (Bialek-Davenet et al., 2015; Foong et al., 2020; Xu et al., 2021). However, we found the 
TABLE 3 | Conjugation frequency of resistant plasmids identified in K. pneumoniae 1678.

\begin{tabular}{|c|c|c|c|c|}
\hline \multirow[t]{2}{*}{ Plasmid } & \multirow[t]{2}{*}{ Resistance gene } & \multirow[t]{2}{*}{ No. of independent determinations } & \multicolumn{2}{|c|}{ Conjugation frequencies } \\
\hline & & & Mean & Range \\
\hline p1678-3 & fOSA3 & 3 & $2.25 \times 10^{-4}$ & $1.97 \times 10^{-4}-2.75 \times 10^{-4}$ \\
\hline p1678-4 & bla NDM-5 & 3 & $1.84 \times 10^{-4}$ & $1.42 \times 10^{-4}-2.37 \times 10^{-4}$ \\
\hline p1678-5 & $m c r-1$ & 3 & $5.41 \times 10^{-3}$ & $2.94 \times 10^{-3}-7.9 \times 10^{-3}$ \\
\hline \multicolumn{5}{|l|}{ Co-transfer of plasmids } \\
\hline Co-transfer of p1678-3 and p1678-5 & fosA3 + mcr-1 & 3 & $1.78 \times 10^{-5}$ & $1.29 \times 10^{-5}-2.77 \times 10^{-5}$ \\
\hline Co-transfer of p1678-4 and p1678-5 & blandM-5 $+m c r-1$ & 3 & $2.25 \times 10^{-5}$ & $1.62 \times 10^{-5}-2.92 \times 10^{-5}$ \\
\hline Co-transfer of $\mathrm{p} 1678-3$ and $\mathrm{p} 1678-4$ and $\mathrm{p} 1678-5$ & fosA3 + bla $_{\mathrm{NDM}-5}+$ mcr-1 & 3 & $9.63 \times 10^{-8}$ & $8.08 \times 10^{-8}-10.38 \times 10^{-8}$ \\
\hline
\end{tabular}

p1678-6 plasmid, harboring mutated tet $(A)$ gene, could not result in tigecycline resistance. According to the genetic comparison of p1678-6 plasmid with other tet(A)-plasmid (tigecyclineresistant), we found p1678-6 plasmid shared low identity with them. Further to analyze the genetic components surrounding tet(A) gene, we found the IS and Tn elements in p1678-6 plasmid also different. As the IS elements sometimes would affect promoter activity (Partridge et al., 2018), we assumed the expression discrepancy of $\operatorname{tet}(A)$ gene in p1687-6 plasmid with other tigecycline-resistant-tet $(A)$ plasmids may be accounted for in this antibiotic-susceptibility phenomenon. Although the Tet(A) protein did not contribute to the tigecycline resistance, we observed the overexpression of the efflux pumps AcrAB and OqxAB in K. pneumoniae 1678, another key factor mediating the tigecycline resistance (Bialek-Davenet et al., 2015; Li et al., 2017). Hence, in this study, the overexpression of RND-type efflux pumps played a crucial role in tigecycline resistance in K. pneumoniae 1678.

Except for resistant to tigecycline, the resistant to Fosfomycin, colistin, carbapenems, and ceftazidime-avibactam in K. pneumoniae 1678 were all associated with the typical resistant plasmids. Both fosA3 and bla $a_{\mathrm{CTX}-\mathrm{M}-55}$ gene were located on p1678-3 plasmid, a typical IncFII plasmid. Previous studies have demonstrated that Fosfomycin-modifying enzymes were present on plasmids belonging to the IncF, IncN, IncA/C, IncHI2, and IncX1 family, whereas IncF was the predominant plasmid incompatibility type (Yang et al., 2019; Zurfluh et al., 2020). Although there existed several FosA variants, FosA3 was the most frequently found Fosfomycin-modifying enzyme worldwide, and many studies have confirmed the dissemination of the fosA3 gene is closely associated with that of the ESBL gene bla $a_{\mathrm{CTX}-\mathrm{M}}$ (Yang et al., 2019), which was consistent with the findings in the p1678-3 plasmid. Our results also showed the p1678-3 plasmid had the completed conservative $35-\mathrm{kb}$ conjugation module of IncF plasmids (Bi et al., 2018), and the self-transmissibility of this plasmid was also confirmed through conjugation assay. As we know, Fosfomycin was a potential regimen for treating extensively drug-resistant bacteria especially for carbapenemase-producing Enterobacteriaceae (CRE), once these isolates uptake the plasmids like p1678-3, the infection treatment would become limited.

$b l a_{\mathrm{NDM}-5}$ plasmid (p1678-4) and $m c r-1$ plasmid (p1678-5) all belong to IncX-type plasmid, holding a smaller size than IncF plasmid, which sometimes makes it easier for movement. p16784 plasmid belongs to IncX3 type, the major vehicle in mediating the dissemination of $b l a_{\mathrm{NDM}}$ (Wu et al., 2019). Several NDM variants have been reported, which commonly contain between 1 and 5 amino acid substitutions compared to NDM-1. Notably, NDM-5 variant, containing the V88L substitution has repeatedly been reported to exhibit enhanced carbapenemase activity (Wu et al., 2019). MICs of ertapenem against strains producing NDM5 were 4 - or 8 -fold higher than those against strains producing NDM-1 (Wu et al., 2019). Moreover, the novel antibiotic agent ceftazidime-avibactam used alone also makes no defense against NDM-5 carbapenemase (Yang et al., 2020). All this information indicated that the spread and pandemic of such IncX3-type p1678-4 plasmid could pose a huge risk to public health.

Like tigecycline, Fosfomycin, and ceftazidime-avibactam, colistin also is a robust antibiotic against infections caused by CRE (Doi, 2019). However, CRKP 1678 also harbored the pandemic IncX4 mcr-1 plasmid (p1678-5), which conferred resistance to colistin. Most plasmids carrying $\mathrm{mcr}-1$ are reported to be transferable, and IncI2 and IncX4 are dominant $\mathrm{mcr}$ - 1 carrying plasmid types. In previous studies, IncI2 and IncX4 plasmids harboring $m c r-1$ were detected in different species of Enterobacteriaceae, owing to the high transfer rate $\left(10^{-1}-10^{-3}\right)$ of $m c r-1$ plasmid (Xiaomin et al., 2020). In this study, we also confirmed the p1678-5 plasmid could be transferred from $K$. pneumoniae to $E$. coli in high in vitro transfer rate.

According to the related genetic analysis and in vitro high conjugation rate, the threat of each single resistant plasmids was verified clearly in K. pneumoniae 1678. Once clinical isolate, especially for CRE, uptake one of these plasmids, the infection treatment would be tougher. Previous studies had reported the co-transfer of resistant element, but it is usually associated with one conjugative plasmid (Costa et al., 2021; Gu et al., 2021; Magi et al., 2021). Previous studies found the $m c r-1$ plasmid also could co-transfer with bla $a_{\mathrm{NDM}}$ plasmid to one recipient, but these plasmids did not transfer from the same donor like the observation in our study (Liang et al., 2021). Notably, in this study we found the mcr-1 plasmid (p16785) could be co-transferred with fos 33 plasmid (p1678-3) or bla $a_{\mathrm{NDM}-5}$ plasmid (p1678-4) in $10^{-5}$ conjugation frequency. Moreover, we also observed these three plasmids could be transferred together, though the transfer rate was not high, the potential risk should be taken seriously. Furthermore, as 


\section{A}

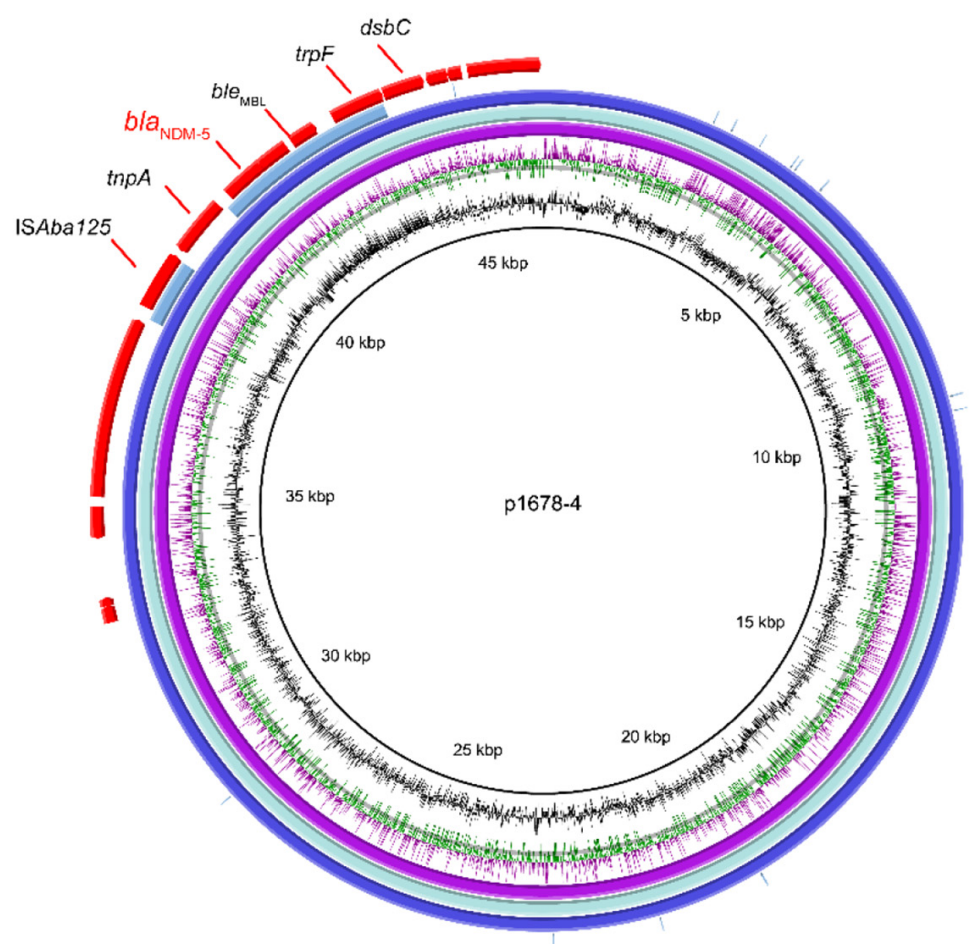

GC Content GC Skew

oc skew(-)

GC Skow $(+)$ p1678-4 (bla NDM-5 $\left._{1}\right)$

$100 \%$ identity

70\% identity

$50 \%$ identity pNDM_MGR194 (bla NOM-S $\left._{-}\right)$

D. $100 \%$ identity

70\% identity

$50 \%$ identity p2B8067 ( bla $_{\text {NOM-7 } 7}$ )

100\% identity

$70 \%$ identity

$50 \%$ identily pNDM-BJ01 (bla $\left.a_{\text {NDM-1 }}\right)$

$100 \%$ identity

$70 \%$ identity

$50 \%$ identity

B

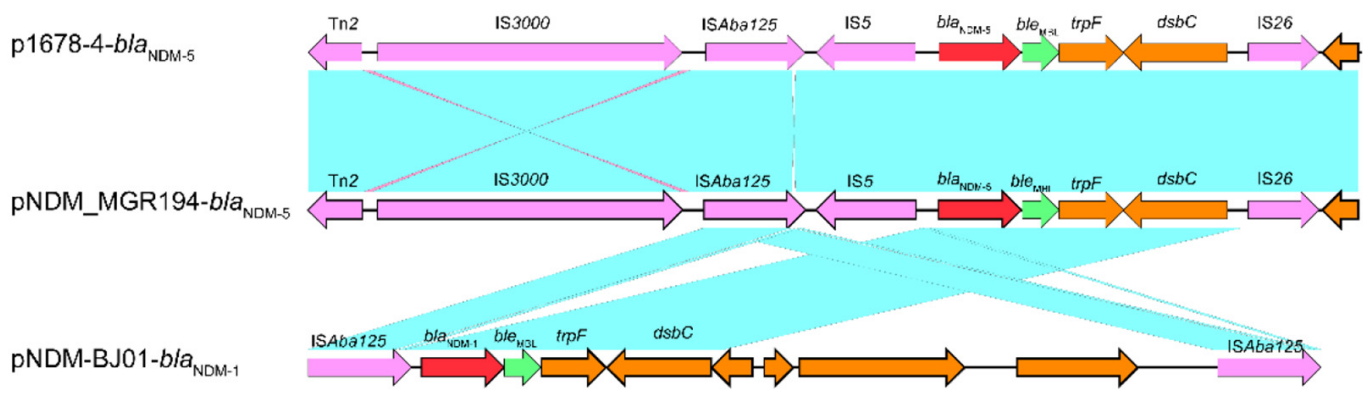

FIGURE 4 | Comparative analysis of pl687-4 plasmids with other reference plasmids. (A) p1678-4 (CP080448) was used as the reference plasmid to perform

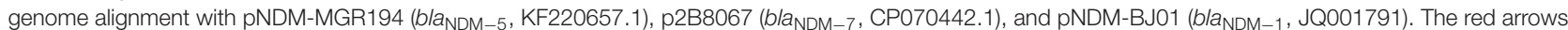

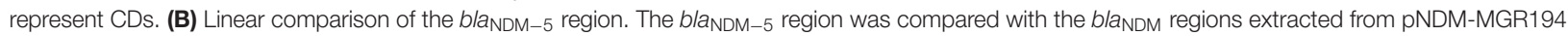
(b/a NDM -5 reference plasmid) and pNDM-BJ01 (classical bla NDM $^{-T n 125}$ transposon).

the overexpression of RND-type efflux transporters (mediating tigecycline resistance) were not rare in K. pneumoniae (BialekDavenet et al., 2015), once these movable resistant plasmids co-transmit to such isolate, like K. pneumoniae 1678 in this study, the therapeutic option would be extremely limited. Tigecycline, Fosfomycin, colistin, carbapenems, and ceftazidime-avibactam are considered as the most effective antibiotics to defend XDR isolates, the co-transferring and co-existing of these typical highrisk plasmids would arise a huge peril to clinical treatment since these antibiotics may all be useless.

The dissemination of resistance genes is not only via plasmids, but also via other mobile structures like transposons and 


\section{A}

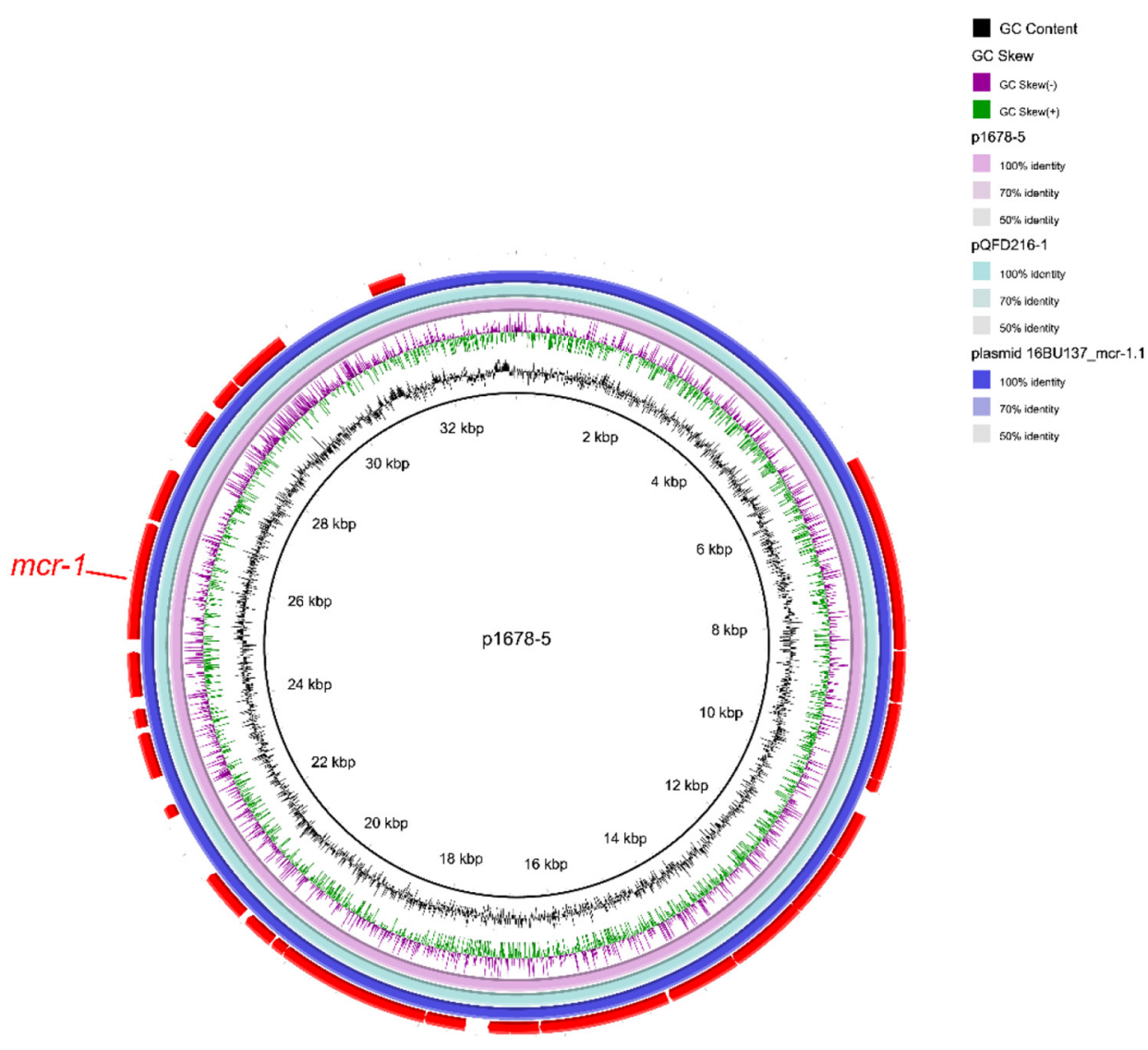

B

p1678-5-mcr-1
K. pneumoniae
PQFD216-1-mcr-1
E.coli
p16BU137-mcr-1
K. pneumoniae

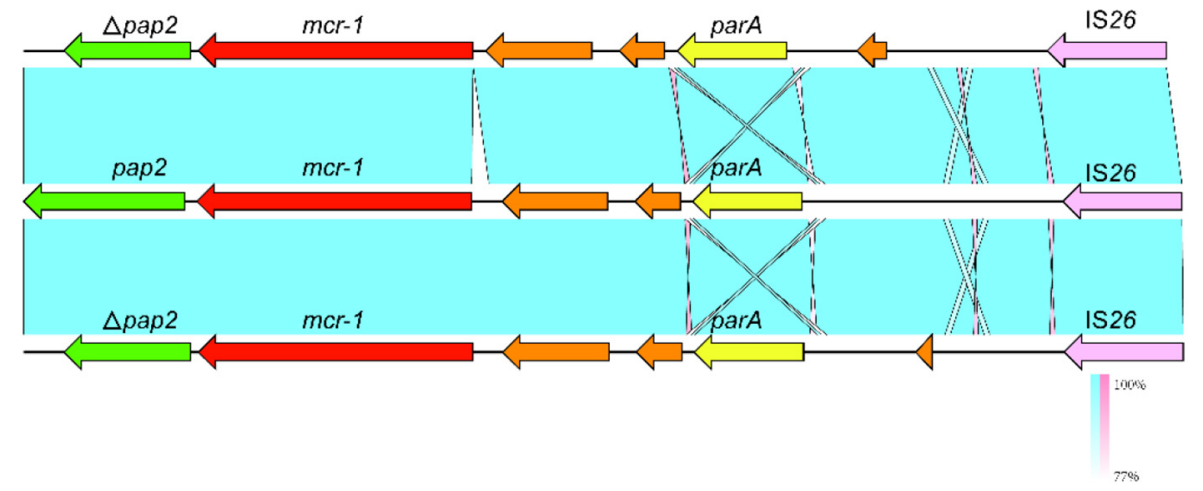

FIGURE 5 | Comparative analysis of pl687-5 plasmids with other reference plasmids. (A) p1678-5 (CP080449) was used as the reference plasmid to perform genome alignment with pQDFD216-1 (CP053212.1, E. coli) and plasmid 16BU137 (MT316509.1, K. pneumoniae). The red arrows represent CDs. (B) Linear comparison of the mcr-1 region. The mcr-1 region was compared with the mcr-1 regions extracted from pQDFD216-1 and pQDFD216-1.

insertion elements. IS26 plays a key role in the dissemination and mobilization of fos A3. This IS26-array forms two composite transposons and several IS26-based transposition units, and both conformations are capable of transposition and exhibit multiple movement modes. In addition to transposition, gene excision and rearrangement of gene modules via homologous recombination between IS26 scattered in the plasmid and/or genome, also drive the evolutionary process of bacteria (Partridge et al., 2018), which could explain the structure of multiple ORF insertions observed in p1678-3 plasmid. The insertion sequence ISAba125 is always upstream of $b l a_{\mathrm{NDM}}$, providing the -35 region of a promoter for the expression of bla $a_{\mathrm{NDM}}$ (Wu et al., 2019). In p1678-4, the Tn125 was truncated by IS26 and IS3000, forming other composite transposons. The mobilization of $b l a_{\mathrm{NDM}}$ associated with IS26 or IS3000 transposons was also common (Wu et al., 2019). These results indicated that although Tn125 transposon 
was interrupted, the movability of $b l a_{\mathrm{NDM}-5}$ remained. Previous studies have indicated that ISApl1 (always associated with the IncI2 plasmid) is a highly active insertion element and a key component required for the mobilization of the gene-cassette containing the $m c r-1$ gene (Xiaomin et al., 2020). However, the ISApl1 was absent in the p1678-5 plasmid, and in all the $\mathrm{mcr}-1$ carrying IncX4-type plasmids, ISApl1 in front of $m c r-1$ was lost (Du et al., 2020). The loss of the composite transposon ISApl1 might increase the stability of the $\mathrm{mor}$ gene in IncX4 plasmids, and promote the widespread dissemination of the $m c r-1$ gene.

In this study, we report the coexistence and co-transferring of FosA3-, NDM-5, and MCR-1-encoding plasmids in a K. pneumoniae isolate. The co-occurrence of fos 33, bla $a_{\mathrm{NDM}-5}$, and $m c r-1$, and the overexpression of RND-type efflux pumps caused 1678 to be highly resistant not only to commonly used antibiotics (e.g., carbapenems, cephalosporins), but also to Fosfomycin, colistin, ceftazidime-avibactam, and tigecycline, which were considered as the last line for defending XDR Gram-negative organisms. Moreover, the high rate of transmission or co-transmission of these plasmids and various mobile elements surrounding resistant genes greatly increased the risk of spread of these resistant phenotypes. The main limitation in this study is we did not apply the conjugation assay between clinical isolates, which means we could not evaluate the dissemination ability of plasmids more accurately, and the K. pneumoniae 1678 did not exhibit any hyper-virulent phenotype. However, future studies are still necessary to evaluate the prevalence of such multi-drug resistant isolates.

\section{REFERENCES}

Ai, W., Zhou, Y., Wang, B., Zhan, Q., Hu, L., Xu, Y., et al. (2021). First report of coexistence of bla (SFO-1) and bla (NDM-1) $\beta$-lactamase genes as well as colistin resistance gene mcr-9 in a transferrable plasmid of a clinical isolate of Enterobacter hormaechei. Front. Microbiol. 12:676113. doi: 10.3389/fmicb.2021. 676113

Bi, D., Zheng, J., Li, J., Sheng, Z., Zhu, X., Ou, H., et al. (2018). In silico typing and comparative genomic analysis of IncFIIK plasmids and insights into the evolution of replicons, plasmid backbones, and resistance determinant profiles. Antimicrob. Agents 62:e00764-18. doi: 10.1128/AAC.007 64-18

Bialek-Davenet, S., Lavigne, J. P., Guyot, K., Mayer, N., Tournebize, R., Brisse, S., et al. (2015). Differential contribution of AcrAB and OqxAB efflux pumps to multidrug resistance and virulence in Klebsiella pneumoniae. J. Antimicrob. Chemother. 70, 81-88. doi: 10.1093/jac/dku340

Chen, L., Mathema, B., Chavda, K. D., Deleo, F. R., Bonomo, R. A., and Kreiswirth, B. N. (2014). Carbapenemase-producing Klebsiella pneumoniae: molecular and genetic decoding. Trends Microbiol. 22, 686-696. doi: 10.1016/j.tim.2014. 09.003

Chen, Y., Liu, Z., Zhang, Y., Zhang, Z., Lei, L., and Xia, Z. (2019). Increasing prevalence of ESBL-producing multidrug resistance Escherichia coli from diseased pets in beijing. China from 2012 to 2017. Front. Microbiol. 10:2852. doi: $10.3389 /$ fmicb.2019.02852

Chopra, I. (2002). New developments in tetracycline antibiotics: glycylcyclines and tetracycline efflux pump inhibitors. Drug Resist Updat. 5, 119-125. doi: 10.1016/s1368-7646(02)00051-1

Costa, A., Figueroa-Espinosa, R., Gaudenzi, F., Lincopan, N., Fuga, B., Ghiglione, B., et al. (2021). Co-occurrence of NDM-5 and RmtB in a clinical isolate of Escherichia coli belonging to CC354 in Latin America. Front. Cell Infect. Microbiol. 11:654852. doi: 10.3389/fcimb.2021.654852

\section{DATA AVAILABILITY STATEMENT}

The datasets presented in this study can be found in online repositories. The names of the repository/repositories and accession number(s) can be found in the article/ Supplementary Material.

\section{AUTHOR CONTRIBUTIONS}

FY conceptualized and designed the study. YZ performed data analysis and interpretation. YZ, WA, and YC wrote the original draft. YG, XCW, BW, LR, YX, HZ, and XYW contributed to the interpretation of data. All authors contributed to the manuscript drafting.

\section{FUNDING}

This study was supported by grants from Shanghai Pulmonary Hospital Development of Discipline-Department of Clinical Laboratory Medicine.

\section{SUPPLEMENTARY MATERIAL}

The Supplementary Material for this article can be found online at: https://www.frontiersin.org/articles/10.3389/fmicb. 2021.811263/full\#supplementary-material

Doi, Y. (2019). Treatment options for carbapenem-resistant gram-negative bacterial infections. Clin. Infect. Dis. 69(Suppl. 7), S565-S575. doi: 10.1093/cid/ ciz830

Du, C., Feng, Y., Wang, G., Zhang, Z., Hu, H., Yu, Y., et al. (2020). Co-occurrence of the mcr-1.1 and mcr-3.7 genes in a multidrug-resistant Escherichia coli isolate from China. Infect. Drug Resist. 13, 3649-3655. doi: 10.2147/IDR.S268787

Fang, L. X., Chen, C., Cui, C. Y., Li, X. P., Zhang, Y., Liao, X. P., et al. (2020). Emerging high-level tigecycline resistance: novel tetracycline destructases spread via the mobile tet(X). Bioessays 42:e2000014. doi: 10.1002/bies. 202000014

Foong, W. E., Wilhelm, J., Tam, H., and Pos, K. M. (2020). Tigecycline efflux in Acinetobacter baumannii is mediated by TetA in synergy with RND-type efflux transporters. J. Antimicrob. Chemoth. 75, 1135-1139. doi: 10.1093/jac/dkaa015

Gu, Y., Lü, Z., Cao, C., Sheng, H., Li, W., Cui, S., et al. (2021). Cunning plasmid fusion mediates antibiotic resistance genes represented by ESBLs encoding genes transfer in foodborne Salmonella. Int. J. Food Microbiol. 355:109336. doi: 10.1016/j.ijfoodmicro.2021.109336

Han, H., Liu, W., Cui, X., Cheng, X., and Jiang, X. (2020). Co-existence of mcr-1 and bla (NDM-5) in an Escherichia coli strain isolated from the pharmaceutical industry. WWTP. Infect Drug Resist. 13, 851-854. doi: 10.2147/IDR.S245047

Hirabayashi, A., Dao, T. D., Takemura, T., Hasebe, F., Trang, L. T., Thanh, N. H., et al. (2021). A transferable incC-incx3 hybrid plasmid cocarrying bla(NDM-4), tet(X), and tmexCD3-toprJ3 confers resistance to carbapenem and tigecycline. mSphere 6:e0059221. doi: 10.1128/mSphere.00592-21

Clinical and Laboratory Standards Institute (2021a). Methods for Dilution Antimicrobial Susceptibility Tests for Bacteria That Grow Aerobically, 11th Edn. Wayne, PA: CLSI.

Clinical and Laboratory Standards Institute (2021b). Performance Standards for Antimicrobial Susceptibility Testing, 28th Edn. Wayne, PA: CLSI.

Krishnaraju, M., Kamatchi, C., Jha, A. K., Devasena, N., Vennila, R., Sumathi, G., et al. (2015). Complete sequencing of an IncX3 plasmid carrying blaNDM-5 
allele reveals an early stage in the dissemination of the blaNDM gene. Indian $\mathrm{J}$. Med. Microbiol. 33, 30-38. doi: 10.4103/0255-0857.148373

Li, R., Han, Y., Zhou, Y., Du, Z., Wu, H., Wang, J., et al. (2017). Tigecycline susceptibility and molecular resistance mechanisms among clinical klebsiella pneumoniae strains isolated during non-tigecycline treatment. Microb. Drug Resist. 23, 139-146. doi: 10.1089/mdr.2015.0258

Liang, Z., Pang, J., Hu, X., Nie, T., Lu, X., Li, X., et al. (2021). Low prevalence of mcr-1 among clinical Enterobacteriaceae isolates and co-transfer of mcr1 and bla(NDM-1) from separate donors. Microb. Drug Resist. 27, 476-484. doi: $10.1089 / \mathrm{mdr} .2020 .0212$

Liu, B. T., and Song, F. J. (2019). Emergence of two Escherichia coli strains coharboring mcr-1 and bla (NDM) in fresh vegetables from China. Infect. Drug Resist. 12, 2627-2635. doi: 10.2147/IDR.S211746

Liu, Y. Y., Wang, Y., Walsh, T. R., Yi, L. X., Zhang, R., Spencer, J., et al. (2016). Emergence of plasmid-mediated colistin resistance mechanism MCR-1 in animals and human beings in China: a microbiological and molecular biological study. Lancet Infect. Dis. 16, 161-168. doi: 10.1016/S1473-3099(15)00424-7

Magi, G., Tontarelli, F., Caucci, S., Sante, L. D., Brenciani, A., Morroni, G., et al. (2021). High prevalence of carbapenem-resistant Klebsiella pneumoniae ST307 recovered from fecal samples in an Italian hospital. Future Microbiol. 16, 703-711. doi: $10.2217 / \mathrm{fmb}-2020-0246$

Mao, J., Liu, W., Wang, W., Sun, J., Lei, S., and Feng, Y. (2018). Antibiotic exposure elicits the emergence of colistin- and carbapenem-resistant Escherichia coli coharboring MCR-1 and NDM-5 in a patient. Virulence 9, 1001-1007. doi: 10.1080/21505594.2018.1486140

Mcconville, T. H., Annavajhala, M. K., Giddins, M. J., Macesic, N., Herrera, C. M., Rozenberg, F. D., et al. (2020). CrrB positively regulates high-level polymyxin resistance and virulence in Klebsiella pneumoniae. Cell Rep. 33:108313. doi: 10.1016/j.celrep.2020.108313

Partridge, S. R., Kwong, S. M., Firth, N., and Jensen, S. O. (2018). Mobile genetic elements associated with antimicrobial resistance. Clin. Microbiol. Rev. 31:e00088-17. doi: 10.1128/CMR.00088-17

Petrosillo, N., Taglietti, F., and Granata, G. (2019). Treatment options for colistin resistant Klebsiella pneumoniae: present and future. J. Clin. Med. 8:934. doi: $10.3390 / \mathrm{jcm} 8070934$

Quan, J., Li, X., Chen, Y., Jiang, Y., Zhou, Z., Zhang, H., et al. (2017). Prevalence of mcr-1 in Escherichia coli and Klebsiella pneumoniae recovered from bloodstream infections in China: a multicentre longitudinal study. Lancet Infect. Dis. 17, 400-410. doi: 10.1016/S1473-3099(16)30528-X

Sun, J., Chen, C., Cui, C., Zhang, Y., Liu, X., Cui, Z., et al. (2019). Plasmid-encoded tet(X) genes that confer high-level tigecycline resistance in Escherichia coli. Nat. Microbiol. 4, 1457-1464. doi: 10.1038/s41564-019-0496-4

Sun, J., Yang, R., Zhang, Q., Feng, Y., Fang, L., Xia, J., et al. (2016). Co-transfer of blaNDM-5 and mcr-1 by an IncX3-X4 hybrid plasmid in Escherichia coli. Nat. Microbiol. 1:176. doi: 10.1038/nmicrobiol.2016.176

Tang, Y., Zhou, Y., Meng, C., Huang, Y., and Jiang, X. (2020). Co-occurrence of a novel VIM-1 and FosA3-encoding multidrug-resistant plasmid and a KPC2-encoding pKP048-like plasmid in a clinical isolate of Klebsiella pneumoniae sequence type 11. Infect. Genet. Evolu. 85:104479. doi: 10.1016/j.meegid.2020. 104479

Tzouvelekis, L. S., Markogiannakis, A., Psichogiou, M., Tassios, P. T., and Daikos, G. L. (2012). Carbapenemases in Klebsiella pneumoniae and other Enterobacteriaceae: an evolving crisis of global dimensions. Clin. Microbiol. Rev. 25, 682-707. doi: 10.1128/CMR.05035-11

Wang, M. G., Yu, Y., Wang, D., Yang, R. S., Jia, L., Cai, D. T., et al. (2021). The emergence and molecular characteristics of new delhi metallo $\beta$-lactamaseproducing Escherichia coli from ducks in guangdong. China. Front. Microbiol. 12:677633. doi: $10.3389 /$ fmicb.2021.677633

Wang, R., Liu, Y., Zhang, Q., Jin, L., Wang, Q., Zhang, Y., et al. (2018). The prevalence of colistin resistance in Escherichia coli and Klebsiella pneumoniae isolated from food animals in China: coexistence of mcr-1 and bla(NDM) with low fitness cost. Int. J. Antimicrob Agents 51, 739-744. doi: 10.1016/j. ijantimicag.2018.01.023

Wu, W., Feng, Y., Tang, G., Qiao, F., Mcnally, A., and Zong, Z. (2019). NDM metallo- $\beta$-lactamases and their bacterial producers in health care settings. Clin. Microbiol. Rev. 32:e00115-18. doi: 10.1128/CMR.00115-18
Xiaomin, S., Yiming, L., Yuying, Y., Zhangqi, S., Yongning, W., and Shaolin, W. (2020). Global impact of mcr-1-positive Enterobacteriaceae bacteria on "one health". Crit. Rev. Microbiol. 46, 565-577. doi: 10.1080/1040841X.2020.18 12510

Xu, J., Zhu, Z., Chen, Y., Wang, W., and He, F. (2021). The plasmid-borne tet(A) gene is an important factor causing tigecycline resistance in ST11 carbapenemresistant Klebsiella pneumoniae under selective pressure. Front. Microbiol. 12:644949. doi: 10.3389/fmicb.2021.644949

Yahav, D., Giske, C. G., Grāmatniece, A., Abodakpi, H., Tam, V. H., and Leibovici, L. (2020). New $\beta$-lactam- $\beta$-lactamase inhibitor combinations. Clin. Microbiol. Rev. 34, e115-e120. doi: 10.1128/CMR.00115-20

Yang, R. S., Feng, Y., Lv, X. Y., Duan, J. H., Chen, J., Fang, L. X., et al. (2016). Emergence of NDM-5- and MCR-1-producing Escherichia coli clones ST648 and ST156 from a single muscovy duck (cairina moschata). Antimicrob Agents Chemother. 60, 6899-6902. doi: 10.1128/AAC.01365-16

Yang, T., Lu, P., and Tseng, S. (2019). Update on fosfomycin-modified genes in Enterobacteriaceae. J. Microbiol. Immunol. Infect. 52, 9-21. doi: 10.1016/j.jmii. 2017.10.006

Yang, Y., Guo, Y., Yin, D., Zheng, Y., Wu, S., Zhu, D., et al. (2020). In vitro activity of cefepime-zidebactam, ceftazidime-avibactam, and other comparators against clinical isolates of Enterobacterales, Pseudomonas aeruginosa, and Acinetobacter baumannii: results from china antimicrobial surveillance network (CHINET) in 2018. Antimicrob Agents Chemother. 65:e01726-20. doi: 10.1128/AAC.01726-20

Yin, D., Dong, D., Li, K., Zhang, L., Liang, J., Yang, Y., et al. (2017). Clonal dissemination of OXA-232 carbapenemase-producing Klebsiella pneumoniae in neonates. Antimicrob Agents Chemother. 61:e00385-17. doi: 10.1128/AAC. 00385- 17

Yuan, J., Wang, X., Shi, D., Ge, Q., Song, X., Hu, W., et al. (2021). Extensive antimicrobial resistance and plasmid-carrying resistance genes in mcr-1positive E. coli sampled in swine, in Guangxi. South China. BMC Vet. Res. 17:86. doi: 10.1186/s12917-021-02758-4

Yusuf, E., Bax, H. I., Verkaik, N. J., and van Westreenen, M. (2021). An update on eight "new" antibiotics against multidrug-resistant gram-negative bacteria. J. Clin. Med. 10:51068. doi: 10.3390/jcm10051068

Zhanel, G. G., Lawson, C. D., Adam, H., Schweizer, F., Zelenitsky, S., Lagacé-Wiens, P. R., et al. (2013). Ceftazidime-avibactam: a novel cephalosporin/ $\beta$-lactamase inhibitor combination. Drugs 73, 159-177. doi: 10.1007/s40265-013-0013-7

Zheng, J., Lin, Z., Sun, X., Lin, W., Chen, Z., Wu, Y., et al. (2018). Overexpression of $\mathrm{OqXAB}$ and $\mathrm{Mac} A \mathrm{~B}$ efflux pumps contributes to eravacycline resistance and heteroresistance in clinical isolates ofKlebsiella pneumoniae. Emerging Microbes Infect. 7, 1-11. doi: 10.1038/s41426-018-0141-y

Zhou, Y., Tang, Y., Fu, P., Tian, D., Yu, L., Huang, Y., et al. (2020). The type I-E CRISPR-cas system influences the acquisition of bla KPC-IncF plasmid in Klebsiella pneumonia. Emerging Microbes Infect. 9, 1011-1022. doi: 10.1080/ 22221751.2020.1763209

Zurfluh, K., Treier, A., Schmitt, K., and Stephan, R. (2020). Mobile fosfomycin resistance genes in Enterobacteriaceae-an increasing threat. Microbiologyopen 9:e1135. doi: 10.1002/mbo3.1135

Conflict of Interest: The authors declare that the research was conducted in the absence of any commercial or financial relationships that could be construed as a potential conflict of interest.

Publisher's Note: All claims expressed in this article are solely those of the authors and do not necessarily represent those of their affiliated organizations, or those of the publisher, the editors and the reviewers. Any product that may be evaluated in this article, or claim that may be made by its manufacturer, is not guaranteed or endorsed by the publisher.

Copyright () 2022 Zhou, Ai, Cao, Guo, Wu, Wang, Rao, Xu, Zhao, Wang and Yu. This is an open-access article distributed under the terms of the Creative Commons Attribution License (CC BY). The use, distribution or reproduction in other forums is permitted, provided the original author $(s)$ and the copyright owner(s) are credited and that the original publication in this journal is cited, in accordance with accepted academic practice. No use, distribution or reproduction is permitted which does not comply with these terms. 